\title{
Energy flow lines for the radiation emitted by a dipole
}

\author{
Jie Shu, Xin Li and Henk F. Arnoldus* \\ Department of Physics and Astronomy, Mississippi State University, Mississippi, USA
}

(Received 16 April 2008; final version received 20 April 2008)

\begin{abstract}
An oscillating electric dipole emits radiation, and the flow of energy in the electromagnetic field is represented by the field lines of the Poynting vector. In the most general state of oscillation the dipole moment vector traces out an ellipse. We have evaluated analytically the field lines of the Poynting vector for the emitted light, and it appears that each field line lies on a cone, which has its axis perpendicular to the plane of the ellipse. The field lines exhibit a vortex structure near the location of the dipole, and they approach a straight line in the far field. It is shown that due to the spiraling of the field lines near the source, the asymptotic limit of a field line is displaced as compared to a ray which would come directly out of the source. Both the spatial extent of the vortex in the near field and the magnitude of the displacement of the image in the far field are of nanoscale dimension.
\end{abstract}

Keywords: dipole radiation; Poynting vector

\section{Introduction}

The most elementary type of electromagnetic radiation is electric dipole radiation. On one hand, when a source of radiation is small compared to the wavelength of the emitted light, the source is in first approximation an electric dipole, and on the other hand, the radiation emitted by atoms and molecules is usually electric dipole radiation. In particular, when an atom is in an excited electronic state it will decay to a lower state and such that the change in magnetic quantum number is $\Delta m=-1$, or 1 , according to the dipole selection rules. The electric and magnetic fields of an electric dipole are well known [1], and the emitted power per unit solid angle can be obtained easily, giving the familiar lobe structure for a linearly-oscillating dipole moment. This radiation pattern changes dramatically when the dipole is located near an interface with a dielectric or metallic medium. The reflected radiation adds to the directly-emitted dipole radiation, leading to an interference pattern, and for a dielectric interface the transmitted radiation is typically strongly peaked around a cone at the critical angle [2-12]. These calculated and measured intensity distributions assume that the radiation is observed in the far field, e.g. at a distance from the source which is much greater than an optical wavelength. When viewed from the far field, the light appears to be emanating from the location of the dipole, and it propagates as a spherical outgoing wave centered around the dipole. The corresponding optical rays

*Corresponding author. Email: arnoldus@ra.msstate.edu 
(the orthogonal trajectories of the wave fronts) are therefore straight lines which appear to come from the location of the source.

In the geometrical optics limit of light propagation certain terms in Maxwell's equations are neglected under the assumption that the wavelength of the light is small compared to other relevant distances. It can then be shown [13] that the light rays in a homogeneous medium are straight lines, irrespective of the source of the radiation, and that the light rays coincide with the field lines of the Poynting vector. In this limit, the field lines of the Poynting vector are straight lines at any distance from the source, and in particular in the near field. However, when the structure of dipole radiation is resolved on the scale of a wavelength, the field lines of the Poynting vector are in general curves [14], and they may exhibit a vortex structure near the location of the dipole. In the far field, the field lines are asymptotically straight, but we shall show that when the direction of the emission of radiation is detected with an accuracy of a wavelength or less, the field lines appear to be displaced as compared to the optical rays. With contemporary near-field optical microscopy techniques, these precise details of a radiation pattern have become amenable to experimental observation $[15,16]$, and such measurements with nanoscopic precision may have an impact on novel imaging devices in microbiology and medical applications.

\section{The Poynting vector for dipole radiation}

When the current density in a localized source of radiation oscillates harmonically with angular frequency $\omega$, it has an electric dipole moment given by

$$
\boldsymbol{d}(t)=\operatorname{Re}\left(\boldsymbol{d} e^{-i \omega t}\right),
$$

where the complex amplitude $\boldsymbol{d}$ is a complex-valued vector, which is determined by the current distribution of the source. It will be assumed that higher order multipole moments of the current density contribute negligibly to the radiation field, when compared to the electric dipole moment contribution. The radiated electric field will also have a harmonic time dependence, and can therefore be written as

$$
\boldsymbol{E}(\boldsymbol{r}, t)=\operatorname{Re}[\boldsymbol{E}(\boldsymbol{r}) \exp (-\mathrm{i} \omega t)],
$$

with $\boldsymbol{E}(\boldsymbol{r})$ the complex amplitude, and the radiated magnetic field $\boldsymbol{B}(\boldsymbol{r}, t)$ can be represented similarly. The complex amplitudes of the electric and magnetic fields of an electric dipole at the origin of coordinates can be represented most compactly as

$$
\begin{gathered}
\boldsymbol{E}(\boldsymbol{r})=\frac{1}{4 \pi \varepsilon_{\mathrm{o}}}\left[k_{\mathrm{o}}^{2} \boldsymbol{d}+(\boldsymbol{d} \cdot \nabla) \nabla\right] g(\boldsymbol{r}), \\
\boldsymbol{B}(\boldsymbol{r})=\frac{\mathrm{i} \omega \mu_{\mathrm{o}}}{4 \pi} \boldsymbol{d} \times \nabla g(\boldsymbol{r}),
\end{gathered}
$$

with $k_{\mathrm{o}}=\omega / c$, and

$$
g(\boldsymbol{r})=\frac{\exp \left(\mathrm{i} k_{\mathrm{o}} r\right)}{r}
$$


is the free-space Green's function for the scalar Helmholtz equation. Working out the derivatives gives explicitly

$$
\begin{gathered}
\boldsymbol{E}(\boldsymbol{r})=\frac{k_{\mathrm{o}}^{3}}{4 \pi \varepsilon_{\mathrm{o}} q}\left\{\boldsymbol{d}-(\boldsymbol{d} \cdot \hat{\boldsymbol{r}}) \hat{\boldsymbol{r}}+[\boldsymbol{d}-3(\boldsymbol{d} \cdot \hat{\boldsymbol{r}}) \hat{\boldsymbol{r}}] \frac{\mathrm{i}}{q}\left(1+\frac{\mathrm{i}}{q}\right)\right\} \exp (\mathrm{i} q), \\
\boldsymbol{B}(\boldsymbol{r})=-\frac{k_{\mathrm{o}}^{3}}{4 \pi \varepsilon_{\mathrm{o}} c q} \boldsymbol{d} \times \hat{\boldsymbol{r}}\left(1+\frac{\mathrm{i}}{q}\right) \exp (\mathrm{i} q),
\end{gathered}
$$

where we have set

$$
q=k_{\mathrm{o}} r
$$

for the dimensionless distance between the dipole and the field point $\boldsymbol{r}$.

The Poynting vector for a time-harmonic field is given by

$$
\boldsymbol{S}(\boldsymbol{r})=\frac{1}{2 \mu_{\mathrm{o}}} \operatorname{Re}\left[\boldsymbol{E}(\boldsymbol{r}) \times \boldsymbol{B}(\boldsymbol{r})^{*}\right]
$$

where terms that oscillate at twice the optical frequency have been dropped since they average to zero. The direction of $\boldsymbol{S}(\boldsymbol{r})$ at the field point $\boldsymbol{r}$ indicates the direction of energy flow, and its magnitude equals the power transported per unit area. The Poynting vector $\boldsymbol{S}(\boldsymbol{r})$ determines a vector field around the dipole, and the field lines of this vector field represent the flow pattern of the radiated energy. When we substitute the right-hand sides of Equations (6) and (7) for the complex amplitudes of the electric and magnetic fields, respectively, we obtain

$$
\boldsymbol{S}(\boldsymbol{r})=\frac{c k_{\mathrm{O}}^{4}}{32 \pi^{2} \varepsilon_{\mathrm{O}} r^{2}}\left\{\left[\boldsymbol{d} \cdot \boldsymbol{d}^{*}-(\hat{\boldsymbol{r}} \cdot \boldsymbol{d})\left(\hat{\boldsymbol{r}} \cdot \boldsymbol{d}^{*}\right)\right] \hat{\boldsymbol{r}}-\frac{2}{q}\left(1+\frac{1}{q^{2}}\right) \operatorname{Im}\left[(\hat{\boldsymbol{r}} \cdot \boldsymbol{d}) \boldsymbol{d}^{*}\right]\right\} .
$$

We then set

$$
\boldsymbol{d}=d_{\mathrm{o}} \boldsymbol{\varepsilon}, \quad d_{\mathrm{o}}>0, \quad \boldsymbol{\varepsilon} \cdot \boldsymbol{\varepsilon}^{*}=1,
$$

for the complex amplitude of the dipole moment and introduce the function

$$
\zeta(\theta, \phi)=1-(\hat{\boldsymbol{r}} \cdot \boldsymbol{\varepsilon})\left(\hat{\boldsymbol{r}} \cdot \boldsymbol{\varepsilon}^{*}\right),
$$

where $\theta$ and $\phi$ are the angles of the field point $\boldsymbol{r}$ in spherical coordinates. This yields the expression

$$
\boldsymbol{S}(\boldsymbol{r})=\frac{3 P_{\mathrm{o}}}{8 \pi r^{2}}\left\{\zeta(\theta, \phi) \hat{\boldsymbol{r}}-\frac{2}{q}\left(1+\frac{1}{q^{2}}\right) \operatorname{Im}\left[(\hat{\boldsymbol{r}} \cdot \boldsymbol{\varepsilon}) \boldsymbol{\varepsilon}^{*}\right]\right\},
$$

for the Poynting vector. Here we have set

$$
P_{\mathrm{o}}=\frac{c k_{\mathrm{o}}^{4}}{12 \pi \varepsilon_{\mathrm{o}}} d_{\mathrm{o}}^{2}
$$


which equals the total radiated power by the electric dipole. For a magnetic dipole, the electric and magnetic fields are different, but it can be shown that the resulting expression (13) for the Poynting vector is the same.

When the vector $\boldsymbol{\varepsilon}$ in Equation (11) is real, as for instance in a $\Delta m=0$ transition in an atom, the dipole moment given by Equation (1) becomes $\boldsymbol{d}(t)=d_{\mathrm{o}} \boldsymbol{\varepsilon} \cos (\omega t)$. This corresponds to a linear dipole moment, oscillating back and forth along the direction of $\varepsilon$, and the Poynting vector becomes

$$
\boldsymbol{S}(\boldsymbol{r})=\frac{3 P_{\mathrm{o}}}{8 \pi r^{2}} \hat{\boldsymbol{r}} \sin ^{2} \alpha,
$$

where $\alpha$ is the angle between vector $\boldsymbol{\varepsilon}$ and the observation direction $\hat{\boldsymbol{r}}$. The Poynting vector is in the radial direction for all field points $\boldsymbol{r}$, and therefore the field lines of $\boldsymbol{S}(\boldsymbol{r})$ are straight lines which run radially outward from the location of the dipole. The power per unit solid angle is proportional to $\sin ^{2} \alpha$, and this gives the common lobe pattern for dipole radiation.

At a large distance from the dipole, the Poynting vector is approximately

$$
\boldsymbol{S}(\boldsymbol{r}) \approx \frac{3 P_{\mathrm{o}}}{8 \pi r^{2}} \zeta(\theta, \phi) \hat{\boldsymbol{r}},
$$

which is in the radial direction. Therefore, in the far field the field lines approach a straight line, and they have the appearance of running from the location of the dipole directly to the far field. A more careful consideration (below) will show that this only holds when spatial variations on the scale of a wavelength in the far field are neglected.

\section{Elliptical dipole moment}

When an atom in an excited electronic state decays to a lower state in a $\Delta m=\mp 1$ transition, the vector $\boldsymbol{\varepsilon}$ in Equation (11) is the spherical unit vector

$$
\boldsymbol{e}_{ \pm 1}=-\frac{1}{2^{1 / 2}}\left( \pm \boldsymbol{e}_{x}+\mathrm{i} \boldsymbol{e}_{y}\right)
$$

The dipole moment $\boldsymbol{d}(t)$ then traces out a circle in the $x y$-plane, and the rotation is positive (counterclockwise when viewed from the positive $z$-axis) for $\boldsymbol{\varepsilon}=\boldsymbol{e}_{1}$ and negative for $\boldsymbol{\varepsilon}=\boldsymbol{e}_{-1}$. In general, however, the complex amplitude $\boldsymbol{d}$ of the dipole moment can be any complex-valued vector. It can then be shown [17] that the most general state of rotation of $\boldsymbol{d}(t)$ is an ellipse. We can then take the plane of this ellipse as the $x y$-plane, and parametrize vector $\boldsymbol{\varepsilon}$ as

$$
\boldsymbol{\varepsilon}=-\frac{1}{\left(\beta^{2}+1\right)^{1 / 2}}\left(\beta \boldsymbol{e}_{x}+\mathrm{i} \boldsymbol{e}_{y}\right),
$$

with $\beta$ real. With Equation (1) this yields

$$
\boldsymbol{d}(t)=-\frac{d_{\mathrm{o}}}{\left(\beta^{2}+1\right)^{1 / 2}}\left[\beta \boldsymbol{e}_{x} \cos (\omega t)+\boldsymbol{e}_{y} \sin (\omega t)\right],
$$




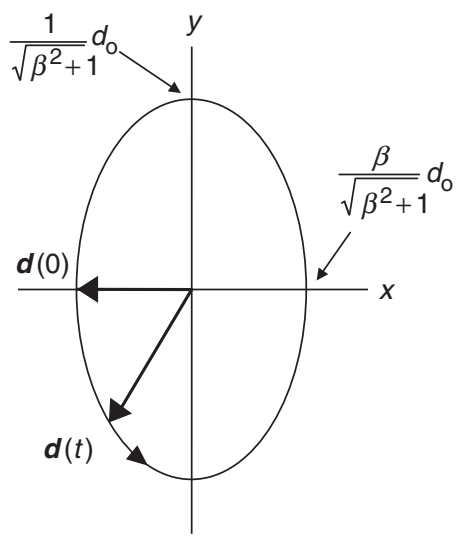

Figure 1. The figure illustrates the most general state of oscillation of an electric dipole moment $\boldsymbol{d}(t)$. As time progresses, vector $\boldsymbol{d}(t)$ traces out an ellipse, and we take the plane of the ellipse as the $x y$-plane. The ellipse is parametrized with $\beta$, as in Equation (19), and the figure shows the dependence on $\beta$ of the semi-major and semi-minor axes. For $\beta=0$, the oscillation becomes linear along the $y$-axis, and for $\beta=1$ the ellipse reduces to a circle. For $\beta>1$ the major axis is along the $x$-axis, and for $\beta \rightarrow \infty$ the oscillation becomes linear along the $x$-axis. For $\beta$ positive, as in the figure, the rotation is counterclockwise and for $\beta$ negative the rotation becomes clockwise.

and this ellipse is shown in Figure 1. For $\beta>0(\beta<0)$ the rotation is positive (negative) and for $\beta= \pm 1$, vector $\boldsymbol{\varepsilon}$ becomes $\boldsymbol{e}_{ \pm 1}$, and the ellipse reduces to a circle. Furthermore, for $\beta=0$ and $\beta \rightarrow \infty$ we recover the case of a linear dipole, oscillating along the $y$-axis and $x$-axis, respectively.

With $\boldsymbol{\varepsilon}$ given by Equation (18), the Poynting vector from Equation (13) becomes

$$
\boldsymbol{S}(\boldsymbol{r})=\frac{3 P_{\mathrm{o}}}{8 \pi r^{2}}\left\{\zeta(\theta, \phi) \hat{\boldsymbol{r}}+\frac{2}{q}\left(1+\frac{1}{q^{2}}\right) \frac{\beta}{\beta^{2}+1} \sin \theta \boldsymbol{e}_{\phi}\right\},
$$

with

$$
\zeta(\theta, \phi)=1-\frac{1}{2} \sin ^{2} \theta\left[1+\frac{\beta^{2}-1}{\beta^{2}+1} \cos (2 \phi)\right] .
$$

The term proportional to $\hat{\boldsymbol{r}}$ in Equation (20) is the same as the right-hand side of Equation (16), so this term corresponds to the far-field solution. The term proportional to $\boldsymbol{e}_{\phi}$ is dominant in the near field, since it is proportional to $r^{-5}$, and it will give rise to a rotation of the field lines around the $z$-axis. For $\beta=0$ and $\beta \rightarrow \infty$ this term vanishes, and the field lines are in the radial direction at any distance from the dipole.

\section{Field lines of the Poynting vector}

Expression (20) for $\boldsymbol{S}(\boldsymbol{r})$ defines a vector field in space, and a field line of $\boldsymbol{S}(\boldsymbol{r})$ is a curve for which at any point along the curve the vector $\boldsymbol{S}(\boldsymbol{r})$ is on its tangent line. Let $\boldsymbol{r}(u)$ be a parametrization of a field line, with $u$ a dummy variable. Since a field line is 
only determined by the direction of $\boldsymbol{S}(\boldsymbol{r})$, and not its magnitude, the field lines are solutions of

$$
\frac{\mathrm{d} \boldsymbol{r}}{\mathrm{d} u}=f(\boldsymbol{r}) \boldsymbol{S}(\boldsymbol{r})
$$

with $f(\boldsymbol{r})$ an arbitrary positive function of $\boldsymbol{r}$. In spherical coordinates $(q, \theta, \phi)$, Equation (22) becomes

$$
\begin{gathered}
\frac{\mathrm{d} q}{\mathrm{~d} u}=k_{\mathrm{o}} f(\boldsymbol{r}) \hat{\boldsymbol{r}} \cdot \boldsymbol{S}(\boldsymbol{r}), \\
q \frac{\mathrm{d} \theta}{\mathrm{d} u}=k_{\mathrm{o}} f(\boldsymbol{r}) \boldsymbol{e}_{\theta} \cdot \boldsymbol{S}(\boldsymbol{r}), \\
q \sin \theta \frac{\mathrm{d} \phi}{\mathrm{d} u}=k_{\mathrm{a}} f(\boldsymbol{r}) \boldsymbol{e}_{\phi} \cdot \boldsymbol{S}(\boldsymbol{r}),
\end{gathered}
$$

which is a set of equations for $q, \theta$ and $\phi$ as functions of $u$. We then take $f(\boldsymbol{r})=8 \pi r^{2} /\left(3 P_{\mathrm{o}} k_{\mathrm{o}}\right)$, and with Equation (20) for $\boldsymbol{S}(\boldsymbol{r})$ we then obtain

$$
\begin{gathered}
\frac{\mathrm{d} q}{\mathrm{~d} u}=\zeta(\theta, \phi), \\
\frac{\mathrm{d} \theta}{\mathrm{d} u}=0, \\
\frac{\mathrm{d} \phi}{\mathrm{d} u}=\frac{2}{q^{2}}\left(1+\frac{1}{q^{2}}\right) \frac{\beta}{\beta^{2}+1} .
\end{gathered}
$$

The solution of Equation (27) is $\theta=\theta_{\mathrm{o}}$, with $\theta_{\mathrm{o}}$ a constant. Therefore, any field line lies on a cone $\theta=\theta_{\mathrm{o}}$. Then in Equation (26) we can replace $\zeta(\theta, \phi)$ by $\zeta\left(\theta_{\mathrm{o}}, \phi\right)$, and when combined with Equation (28) this yields

$$
\frac{\mathrm{d} \phi}{\mathrm{d} q}=\frac{2}{q^{2}}\left(1+\frac{1}{q^{2}}\right) \frac{1}{\zeta\left(\theta_{\mathrm{o}}, \phi\right)} \frac{\beta}{\beta^{2}+1},
$$

which is an ordinary first-order nonlinear differential equation for $\phi(q)$. We shall solve this equation in the next section.

\section{Solution for the field lines}

A point on a field line has spherical coordinates $(q, \theta, \phi)$. Along a field line, $\theta=\theta_{\mathrm{o}}$, and the coordinates $\phi$ and $q$ are related by Equation (29). This equation is separable, and with the explicit form (21) for $\zeta\left(\theta_{0}, \phi\right)$ we find as integral

$$
\left(1-\frac{1}{2} \sin ^{2} \theta_{\mathrm{o}}\right) \phi-\frac{1}{4} \sin ^{2} \theta_{\mathrm{o}} \sin (2 \phi) \frac{\beta^{2}-1}{\beta^{2}+1}=-\frac{2 \beta}{\beta^{2}+1} \frac{1}{q}\left(1+\frac{1}{3 q^{2}}\right)+C .
$$


In order to determine the integration constant $C$, we first notice that $\mathrm{d} \phi / \mathrm{d} q$ is positive (negative) for $\beta$ positive (negative), since the function $\zeta\left(\theta_{0}, \phi\right)$ is non-negative. Therefore, $\phi$ increases (decreases) monotonically with $q$ for $\beta$ positive (negative). On the other hand, we have $\mathrm{d} \phi / \mathrm{d} q \rightarrow 0$ for $q \rightarrow \infty$, and consequently $\phi$ reaches a final value. When we set $\phi_{\mathrm{o}}=\phi(q \rightarrow \infty)$, we find from Equation (30)

$$
C=\left(1-\frac{1}{2} \sin ^{2} \theta_{\mathrm{o}}\right) \phi_{\mathrm{o}}-\frac{1}{4} \sin ^{2} \theta_{\mathrm{o}} \sin \left(2 \phi_{\mathrm{o}}\right) \frac{\beta^{2}-1}{\beta^{2}+1},
$$

for the integration constant. We then obtain

$$
\begin{aligned}
(1 & \left.-\frac{1}{2} \sin ^{2} \theta_{\mathrm{o}}\right)\left(\phi-\phi_{\mathrm{o}}\right)-\frac{1}{4} \sin ^{2} \theta_{\mathrm{o}}\left[\sin (2 \phi)-\sin \left(2 \phi_{\mathrm{o}}\right)\right] \frac{\beta^{2}-1}{\beta^{2}+1} \\
& =-\frac{2 \beta}{\beta^{2}+1} \frac{1}{q}\left(1+\frac{1}{3 q^{2}}\right),
\end{aligned}
$$

relating implicitly $\phi$ and $q$ along a field line. Apart from $\beta$, the free parameters are $\theta_{\mathrm{o}}$ and $\phi_{\mathrm{o}}$. A field line lies on the cone $\theta=\theta_{\mathrm{o}}$, and the values of $\phi$ along a field line are in the range

$$
\begin{gathered}
-\infty<\phi<\phi_{\mathrm{o}}, \quad \beta>0, \\
\phi_{\mathrm{o}}<\phi<\infty, \quad \beta<0,
\end{gathered}
$$

as follows from the arguments above.

Equation (32) can be solved explicitly, provided we consider $\phi$ as the independent variable rather than $q$. For a given $\phi$, Equation (32) is a cubic equation in $q$, and the solution is

$$
q(\phi)=\frac{1}{\left[\left(1+\frac{1}{4} A(\phi)^{2}\right)^{1 / 2}+\frac{1}{2} A(\phi)\right]^{1 / 3}-\left[\left(1+\frac{1}{4} A(\phi)^{2}\right)^{1 / 2}-\frac{1}{2} A(\phi)\right]^{1 / 3}},
$$

where we have introduced the function

$$
A(\phi)=\frac{3}{8}\left(\beta-\frac{1}{\beta}\right) \sin ^{2} \theta_{\mathrm{o}}\left[\sin (2 \phi)-\sin \left(2 \phi_{\mathrm{o}}\right)\right]-\frac{3}{2}\left(\beta+\frac{1}{\beta}\right)\left(1-\frac{1}{2} \sin ^{2} \theta_{\mathrm{o}}\right)\left(\phi-\phi_{\mathrm{o}}\right) .
$$

The dimensionless Cartesian coordinates of a point on a field line are therefore parametrized by

$$
\begin{gathered}
\bar{x}=q(\phi) \sin \theta_{\mathrm{o}} \cos \phi, \\
\bar{y}=q(\phi) \sin \theta_{\mathrm{o}} \sin \phi, \\
\bar{z}=q(\phi) \cos \theta_{\mathrm{O}},
\end{gathered}
$$

with $\bar{x}=k_{\mathrm{o}} x, \bar{y}=k_{\mathrm{o}} y$ and $\bar{z}=k_{\mathrm{o}} z$, and the parameter $\phi$ is chosen as in Equation (33) or (34), depending on the sign of $\beta$.

Figure 2 shows several field lines for different values of $\theta_{\mathrm{o}}$, and for each we have $\beta=1$ and $\phi_{\mathrm{o}}=\pi / 2$. Each field line lies on a cone with its axis as the $z$-axis, and the field lines turn 


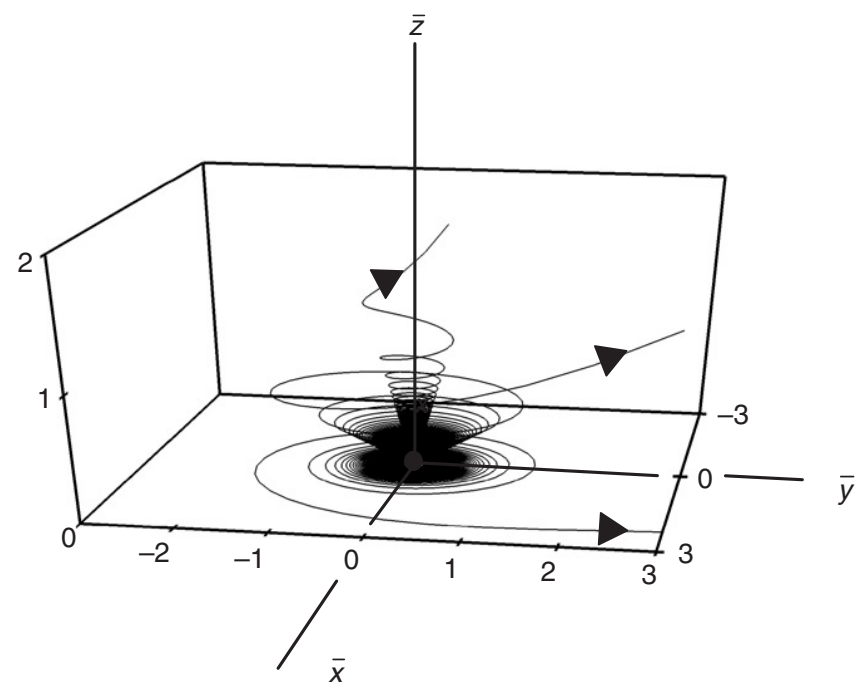

Figure 2. Shown are three field lines of the Poynting vector for $\beta=1$ and $\phi_{\mathrm{o}}=\pi / 2$, with $\theta_{\mathrm{o}}=\pi / 8$, $3 \pi / 8$ and $\pi / 2$. Each field line lies on the corresponding cone, and leaves in the positive $y$-direction.

around the $z$-axis with a positive orientation. Asymptotically, the field lines run into the direction of the positive $y$-axis since we have $\phi_{\mathrm{o}}=\pi / 2$. For the dimensionless coordinates we have $\bar{x}=k_{\mathrm{o}} x$, etc., so a dimensionless distance of $2 \pi$ corresponds to one optical wavelength. We then see from the figure that the spatial extent of this optical vortex is a fraction of a wavelength. For optical radiation, with a wavelength of several hundred nanometers, the vortex manifests itself on a scale of less than $100 \mathrm{~nm}$. With contemporary high-precision nanoscale experimental techniques, such a vortex should be accessible to detection. For the field lines in the region $z>0$ in Figure 3 we have chosen $\beta=1$ and $\theta_{\mathrm{o}}=\pi / 4$, and each field line has a different value of $\phi_{\mathrm{o}}$. For the field lines below the $x y$-plane, we have $\beta=1$ and the values of $\phi_{\mathrm{o}}$ are the same as for the region $z>0$, but $\theta_{\mathrm{o}}$ is equal to $3 \pi / 4$. The orientation of the field lines with respect to the $z$-axis is the same in $z>0$ and in $z<0$. The dependence of the vortex structure on the value of $\beta$ is illustrated in Figure 4 for $\theta_{\mathrm{o}}=\pi / 4$ and $\phi_{\mathrm{o}}=\pi / 2$. For $\beta=1$, Figure $4(a)$, shows a similar vortex as in Figures 2 and 3. When the value of $\beta$ decreases, as in Figures $4(b)$ and $(c)$, we find that the structure of the vortex remains similar, but its spatial extent diminishes. For $\beta \rightarrow 0$ the field lines of the Poynting vector should become straight lines, running from the site of the dipole to the far field, and Figure 4 shows that this transition occurs through a decreasing size of the optical vortex.

\section{Asymptotic limit of the field lines}

Every field line of the Poynting vector approaches a straight line in the far field, which is reminiscent of the optical rays picture for the emission of radiation. Due to the rotation of the field lines near the location of the source, however, these straight lines do not appear to come exactly from the site of the dipole, as depicted in Figure 5. In order to obtain the 


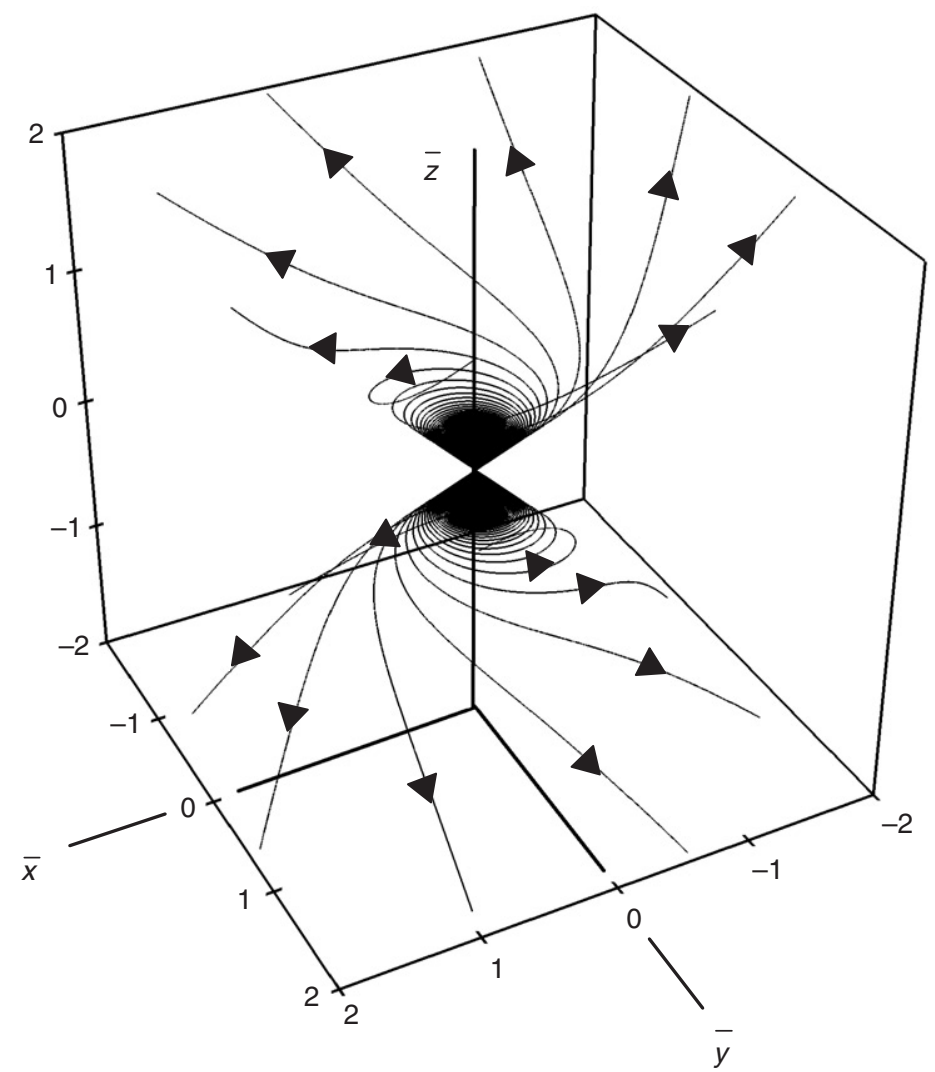

Figure 3. The figure shows field lines of the Poynting for $\beta=1$ and $\theta_{\mathrm{o}}=\pi / 4$ and $3 \pi / 4$, and for a variety of values of $\phi_{\mathrm{o}}$. The orientation of the field lines in both $z>0$ and $z<0$ is the same as the direction of rotation of the dipole moment (positive).

asymptotic limit of the field lines, we consider Equation (29) for $\mathrm{d} \phi / \mathrm{d} q$. For $q$ large, we can expand $\phi(q)$ in an asymptotic series as

$$
\phi(q)=\phi_{\mathrm{o}}+\frac{\alpha_{1}}{q}+\frac{\alpha_{2}}{q^{2}}+\cdots .
$$

For the function $\zeta\left(\theta_{\mathrm{o}}, \phi\right)$ on the right-hand side of Equation (29) we obtain $\zeta\left(\theta_{\mathrm{o}}, \phi\right)=$ $\zeta\left(\theta_{\mathrm{o}}, \phi_{\mathrm{o}}\right)+\mathcal{O}(1 / q)$, and the expansion of Equation (29) becomes

$$
\frac{\mathrm{d} \phi}{\mathrm{d} q}=\frac{1}{q^{2}} Y\left(\theta_{\mathrm{o}}, \phi_{\mathrm{o}} ; \beta\right)+\mathcal{O}\left(\frac{1}{q^{3}}\right)
$$

where we have introduced the abbreviation

$$
Y\left(\theta_{\mathrm{o}}, \phi_{\mathrm{o}} ; \beta\right)=\frac{1}{\zeta\left(\theta_{\mathrm{o}}, \phi_{\mathrm{o}}\right)} \frac{2 \beta}{\beta^{2}+1} .
$$



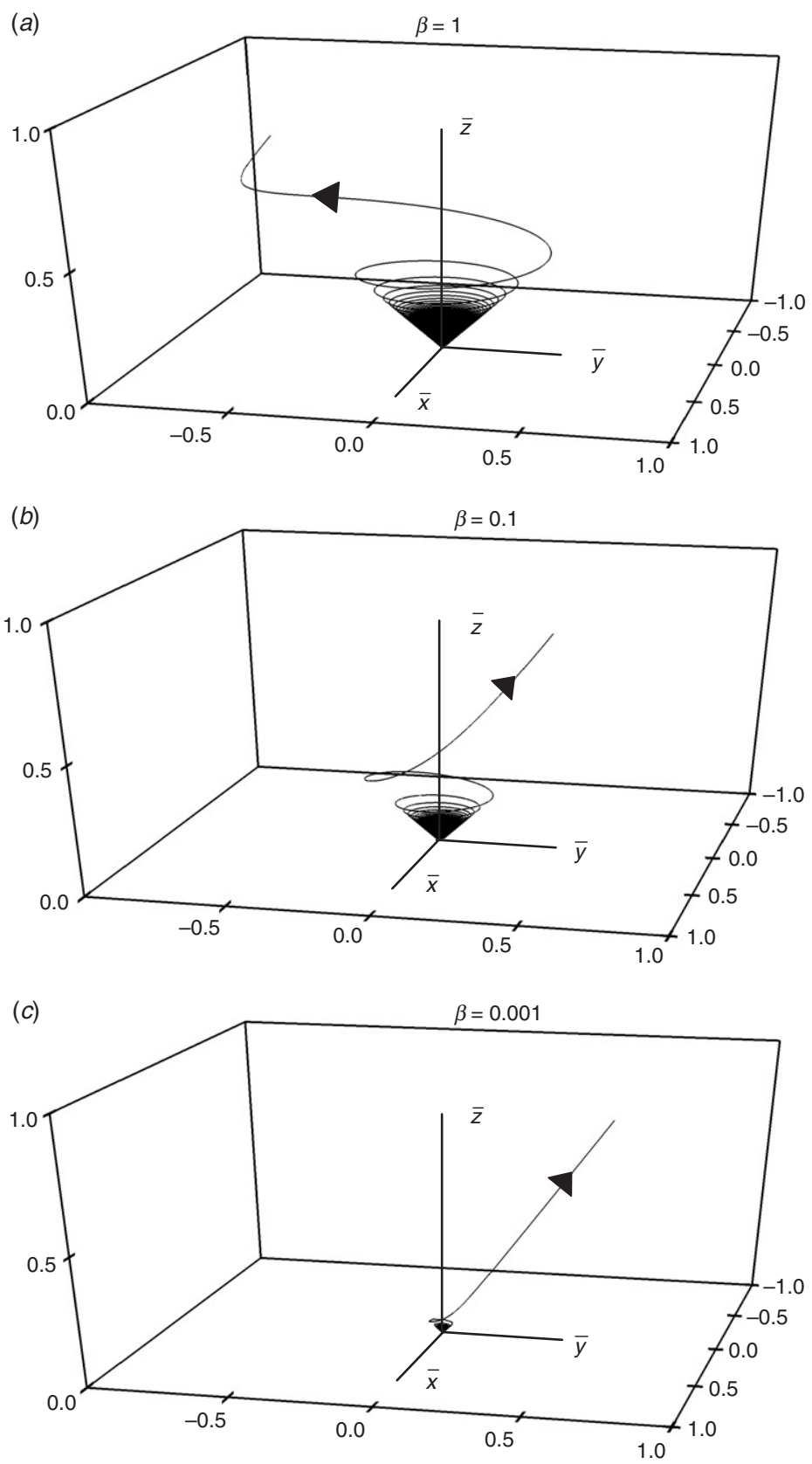

Figure 4. Parts $(a)-(c)$ illustrate the dependence of the field lines on $\beta$. The observation direction is $\left(\theta_{\mathrm{o}}, \phi_{\mathrm{o}}\right)=(\pi / 4, \pi / 2)$ for each. (a) For which $\beta=1$, corresponds to a circular dipole. When $\beta$ decreases, the dipole approaches a linear dipole, oscillating along the $y$-axis, for which the field lines are straight, and run radially outward. It appears that this limit is reached in such a way that the spatial extent of the vortex reduces, until it reaches a point for $\beta \rightarrow 0$, as can be seen from (c). 


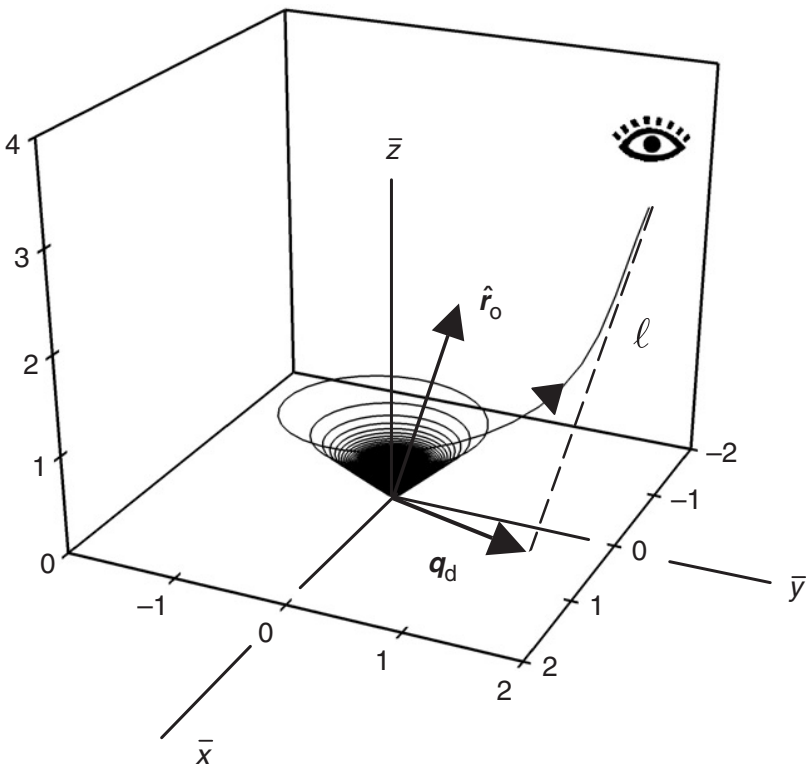

Figure 5. A field line approaches asymptotically a line $\ell$ at a large distance. When the radiation is detected by an observer in the far field, the field line appears to come from a point in the $x y$-plane with position vector $\boldsymbol{q}_{\mathrm{d}}$. Therefore, the source of the radiation appears to be displaced over vector $\boldsymbol{q}_{\mathrm{d}}$. The figure illustrates this displacement for a circular dipole with $\beta=1$, and observation direction $\left(\theta_{\mathrm{o}}, \phi_{\mathrm{o}}\right)=(\pi / 4,0.95 \pi)$.

Integration of Equation (41) then yields the first two terms of the asymptotic series

$$
\phi(q)=\phi_{\mathrm{o}}-\frac{1}{q} Y\left(\theta_{\mathrm{o}}, \phi_{\mathrm{o}} ; \beta\right)+\cdots .
$$

The dimensionless Cartesian coordinates for a point on a field line are given by Equations (37)-(39), in which $q$ is considered a function of $\phi$. We now view $\phi$ to be a function of $q$, and we expand $\cos \phi$ and $\sin \phi$ in Equations (37) and (38) for $q$ large by means of Equation (43). This gives

$$
\begin{aligned}
& \cos \phi(q)=\cos \phi_{\mathrm{o}}+\frac{1}{q} Y\left(\theta_{\mathrm{o}}, \phi_{\mathrm{o}} ; \beta\right) \sin \phi_{\mathrm{o}}+\cdots, \\
& \sin \phi(q)=\sin \phi_{\mathrm{o}}-\frac{1}{q} Y\left(\theta_{\mathrm{o}}, \phi_{\mathrm{o}} ; \beta\right) \cos \phi_{\mathrm{o}}+\cdots .
\end{aligned}
$$

In Equations (37) and (38) the factors $\cos \phi$ and $\sin \phi$ are multiplied by $q$, and therefore the second terms on the right-hand sides of Equations (44) and (45) become a constant, independent of $q$. The higher-order terms vanish for $q \rightarrow \infty$, and therefore the asymptotic limit for the dimensionless Cartesian coordinates becomes

$$
\bar{x}=\sin \theta_{\mathrm{o}}\left[\lambda \cos \phi_{\mathrm{o}}+Y\left(\theta_{\mathrm{o}}, \phi_{\mathrm{o}} ; \beta\right) \sin \phi_{\mathrm{o}}\right],
$$




$$
\begin{gathered}
\bar{y}=\sin \theta_{\mathrm{o}}\left[\lambda \sin \phi_{\mathrm{o}}-Y\left(\theta_{\mathrm{o}}, \phi_{\mathrm{o}} ; \beta\right) \cos \phi_{\mathrm{o}}\right], \\
\bar{z}=\lambda \cos \theta_{\mathrm{o}},
\end{gathered}
$$

where we have replaced $q$ by $\lambda$, since this free parameter does not have the significance of the dimensionless distance to the origin anymore in the asymptotic limit. When we let $-\infty<\lambda<\infty$, Equations (46)-(48) represent a straight line $\ell$, which is the asymptote of the corresponding field line of the Poynting vector. When we set

$$
\boldsymbol{q}_{\mathrm{d}}=Y\left(\theta_{\mathrm{o}}, \phi_{\mathrm{o}} ; \beta\right) \sin \theta_{\mathrm{o}}\left(\boldsymbol{e}_{x} \sin \phi_{\mathrm{o}}-\boldsymbol{e}_{y} \cos \phi_{\mathrm{o}}\right),
$$

for given $\left(\theta_{\mathrm{o}}, \phi_{\mathrm{o}}\right)$, then the equation for the line $\ell$ can be written as

$$
\ell: \boldsymbol{q}=\lambda \hat{\boldsymbol{r}}_{\mathrm{o}}+\boldsymbol{q}_{\mathrm{d}},
$$

with $\boldsymbol{q}=k_{\mathrm{o}} \boldsymbol{r}$ the dimensionless position vector for a point on the line $\ell$, and $\hat{\boldsymbol{r}}_{\mathrm{O}}$ the radial unit vector into the direction of observation $\left(\theta_{\mathrm{o}}, \phi_{\mathrm{o}}\right)$. The intersection between the line $\ell$ and the $x y$-plane follows by setting $\lambda=0$, and therefore we see that vector $\boldsymbol{q}_{\mathrm{d}}$ is the virtual displacement of the source in the $x y$-plane, when viewed from the far field. This result is illustrated in Figure 5. It also follows from Equation (49) that $\boldsymbol{q}_{\mathrm{d}} \cdot \hat{\boldsymbol{r}}_{\mathrm{o}}=0$, and therefore the displacement $\boldsymbol{q}_{d}$ is perpendicular to the direction of observation $\hat{\boldsymbol{r}}_{\mathrm{o}}$.

\section{Displacement in the far field}

When a field line is observed in the far field, it appears to come from the point with position vector $\boldsymbol{q}_{\mathrm{d}}$ in the $x y$-plane, as shown in Figure 5. From a different point of view, the observation plane of an observer in the far field, located at angular position $\left(\theta_{\mathrm{o}}, \phi_{\mathrm{o}}\right)$, is a plane perpendicular to $\hat{\boldsymbol{r}}_{\mathrm{O}}$, at a large distance from the source. When a field line would run straight from the source to the observer, it would intersect the observation plane at the local origin of coordinates $\mathcal{O}^{\prime}$, as shown in Figure 6, but due to the rotation of the field lines near the source the field line intersects this plane at a different point. At a large

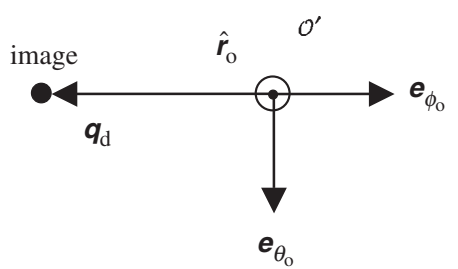

Figure 6. The figure shows the observation plane for an observer located in the direction $\hat{r}_{\mathrm{o}}$ with respect to the source. The plane is perpendicular to $\hat{\boldsymbol{r}}_{\mathrm{O}}$ and far away from the source. If a field line would be a straight line (ray) from the source to the far field, it would intersect the observation plane at the origin $\mathcal{O}^{\prime}$. Due to the rotation of the field line near the source, the intersection point, which is the location of the image, is displaced over vector $\boldsymbol{q}_{\mathrm{d}}$ in the observation plane. For a positive $\beta$ the rotation near the source is in the positive direction with respect to the $z$-axis, and this leads to a displacement in the $-\boldsymbol{e}_{\phi_{\mathrm{o}}}$ direction, as shown. 
distance, this point is the same as the intersection of the plane with the line $\ell$. The displacement vector $\boldsymbol{q}_{\mathrm{d}}$ from Equation (49) can be written as

$$
\boldsymbol{q}_{\mathrm{d}}=-\boldsymbol{e}_{\phi_{\mathrm{o}}} Y\left(\theta_{\mathrm{o}}, \phi_{\mathrm{o}} ; \beta\right) \sin \theta_{\mathrm{o}},
$$

with $\boldsymbol{e}_{\phi_{\mathrm{o}}}$ the local unit vector $\boldsymbol{e}_{\phi}$ in a spherical coordinate system, and evaluated at the observation angle $\phi_{\mathrm{o}}$. From Equations (50) and (51) it then follows that the displacement of this intersection point with respect to the local origin of coordinates is also given by $\boldsymbol{q}_{\mathrm{d}}$, since vector $\boldsymbol{q}_{\mathrm{d}}$ is a vector in the observation plane. Therefore, the apparent displacement of the image in the far field is the same as the virtual displacement of the source in the $x y$-plane.

The displacement in the far field depends on the observation direction $\left(\theta_{\mathrm{o}}, \phi_{\mathrm{o}}\right)$, and on the parameter $\beta$ of the ellipse. For $\beta>0$, the displacement is in the $-\boldsymbol{e}_{\phi_{\mathrm{o}}}$ direction, as in Figure 6 , and for $\beta<0$ the displacement is in the $\boldsymbol{e}_{\phi_{\mathrm{o}}}$ direction. The magnitude of the displacement is

$$
q_{\mathrm{d}}=\frac{2|\beta|}{\beta^{2}+1} \frac{\sin \theta_{\mathrm{o}}}{\zeta\left(\theta_{\mathrm{o}}, \phi_{\mathrm{o}}\right)},
$$

with $\zeta\left(\theta_{\mathrm{o}}, \phi_{\mathrm{o}}\right)$ given by Equation (21). For $\theta_{\mathrm{o}}=0$ or $\theta_{\mathrm{o}}=\pi$ we have $\zeta\left(\theta_{\mathrm{o}}, \phi_{\mathrm{o}}\right)=1$ and $q_{\mathrm{d}}=0$. When a field line is observed along the $z$-axis, the displacement is zero. We see from Figure 2 that in the $z$-direction a field line swirls around the $z$-axis and stays close to the $z$-axis, which results in a vanishing displacement in the far field. For a given $\beta$ and $\phi_{\mathrm{o}}$ we find from Equations (52) and (21) that $q_{\mathrm{d}}$ is maximum for $\theta_{\mathrm{o}}=\pi / 2$, so for an observation direction in the $x y$-plane. When considering the dependence on $\phi_{\mathrm{o}}$, we find from Equation (21) that $q_{d}$ is maximum for $\cos \left(2 \phi_{\mathrm{o}}\right)=1$ when $|\beta|>1$, and for $\cos \left(2 \phi_{\mathrm{o}}\right)=-1$ when $|\beta|<1$. This corresponds to $\phi_{\mathrm{o}}=0$ or $\pi$ and $\phi_{\mathrm{o}}= \pm \pi / 2$, respectively. From Figure 1 we then see that both cases correspond to an observation direction along the major axis of the ellipse. When viewed in this direction, the magnitude of the displacement is given by

$$
q_{\mathrm{d}}= \begin{cases}2|\beta|, & |\beta|>1, \\ \frac{2}{|\beta|}, & |\beta|<1,\end{cases}
$$

which is the maximum value of $q_{\mathrm{d}}$, given $\beta$. For a circular dipole we have $|\beta|=1$, and the maximum dimensionless displacement is $q_{\mathrm{d}}=2$, corresponding to a distance $r_{\mathrm{d}}=\lambda / \pi$, with $\lambda$ the wavelength of the radiation. When the eccentricity of the ellipse increases, the value of $q_{\mathrm{d}}$ along the major axis increases. For $\beta \rightarrow \infty(\beta \rightarrow 0)$, the oscillation of the dipole becomes linear along the $x$-axis ( $y$-axis), and from Equation (53) it follows that in this limit the maximum displacement grows without bounds. This is due to the fact that along the major axis we have $\zeta\left(\theta_{\mathrm{o}}, \phi_{\mathrm{o}}\right) \rightarrow 0$ for both $\beta \rightarrow \infty$ and $\beta \rightarrow 0$, resulting in a division by a small number in Equation (52). On the other hand, it follows from Equation (16) that $\zeta(\theta, \phi)$ is proportional to the radiated power per unit solid angle in the direction $(\theta, \phi)$. We conclude that in the limit of a linear dipole the displacement is maximum for the direction into which the emitted power vanishes. This phenomenon is illustrated in Figure 7. 


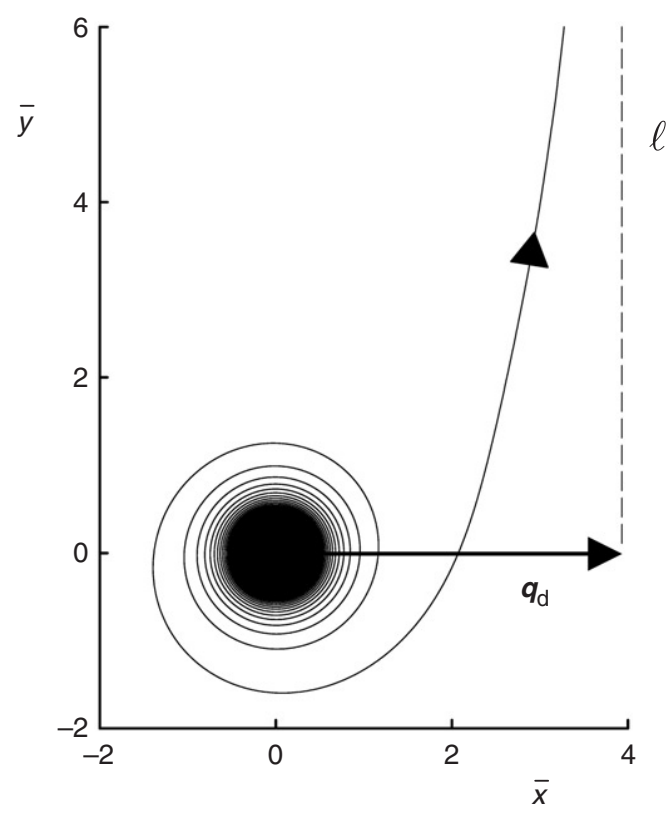

Figure 7. When a field line is observed along the major axis of the ellipse traced out by the rotating dipole moment, the displacement of the image in the $x y$-plane increases with increasing eccentricity of the ellipse. Shown is such a field line for $\beta=0.5$. The displacement vector is along the positive $x$-axis, and its magnitude is equal to $q_{\mathrm{d}}=4$. For a circular dipole, with $|\beta|=1$, the maximum displacement is $q_{\mathrm{d}}=2$.

\section{Conclusions}

In its most general state of oscillation, an electric dipole moment of a source of radiation traces out an ellipse in a plane, taken to be the $x y$-plane. The field lines of the Poynting vector of the emitted electromagnetic field represent the direction of energy flow, and we have obtained an analytical solution for these field lines. It was found that for a given observation direction $\left(\theta_{\mathrm{o}}, \phi_{\mathrm{o}}\right)$ in the far field, the corresponding field line lies entirely on the cone specified by the polar angle $\theta_{\mathrm{o}}$. Near the location of the dipole the field lines have a vortex structure, in which each field line swirls around the $z$-axis numerous times. In the far field, each field line approaches asymptotically a straight line, resembling an optical ray. The parameter equation of this line is given by Equation (50) for a given observation direction $\left(\theta_{\mathrm{o}}, \phi_{\mathrm{o}}\right)$. This line does not go through the origin of coordinates, where the dipole is located, and therefore it appears that the position of the dipole in the $x y$-plane is shifted. This apparent displacement of the source is represented by the position vector $\boldsymbol{q}_{\mathrm{d}}$, given by Equation (49). The magnitude of vector $\boldsymbol{q}_{\mathrm{d}}$ is of the same order as the spatial extent of the vortex near the source, which is of sub-wavelength dimension. However, when the radiation is observed along the major axis of the ellipse, the magnitude of the displacement increases with increasing eccentricity of the ellipse. When a field line is viewed from a location in the far field, the image point is displaced by the same vector $\boldsymbol{q}_{\mathrm{d}}$, with respect to the origin of coordinates in the observation plane. In this fashion, the nanoscale 
structure of the radiation pattern near the source is reflected in a measurable, although small, effect in the far field.

\section{References}

[1] Jackson, J.D. Classical Electrodynamics, 3rd ed.; Wiley: New York, 1999; p 411.

[2] Strutt, M.J.O. Ann. Phys. 1929, 393, 721-750.

[3] Strutt, M.J.O. Ann. Phys. 1929, 393, 751-772.

[4] Drexhage, K.H. In Progress in Optics; Wolf, E., Ed.; Amsterdam: North-Holland, 1974; Vol. XII, pp 163-232.

[5] Lukosz, W.; Kunz, R.E. Opt. Commun. 1977, 20, 195-199.

[6] Lukosz, W.; Kunz, R.E. J. Opt. Soc. Am. 1977, 67, 1607-1615.

[7] Lukosz, W.; Kunz, R.E. J. Opt. Soc. Am. 1977, 67, 1615-1619.

[8] Chance, R.R.; Prock, A.; Silbey, R. Adv. Chem. Phys. 1978, 39, 1-65.

[9] Sipe, J.E. Surf. Sci. 1981, 105, 489-504.

[10] Ford, G.W.; Weber, W.H. Surf. Sci. 1981, 109, 451-481.

[11] Ford, G.W.; Weber, W.H. Phys. Rep. 1984, 113, 195-287.

[12] Arnoldus, H.F.; Foley, J.T. J. Opt. Soc. A 2004, 21, 1109-1117.

[13] Born, M.; Wolf, E. Principles of Optics, 6th ed.; Pergamon: Oxford, 1980; Chapter 3.

[14] Arnoldus, H.F.; Foley, J.T. Opt. Commun. 2004, 231, 115-128.

[15] Lee, K.G.; Kihm, H.W.; Kihm, J.E.; Choi, W.J.; Kim, H.; Ropers, C.; Park, D.J.; Yoon, Y.C.; Choi, S.B.; Woo, D.H., et al. Nature Photonics. 2007, 1, 53-56.

[16] Ohdaira, Y.; Inoue, T.; Hori, H.; Kitahara, K. Opt. Express. 2008, 16, 2915-2921.

[17] Lindell, I.V. Methods for Electromagnetic Field Analysis; Oxford University Press: Oxford, 1992; Section 1.4. 